\title{
Gearbox fault diagnosis using ensemble empirical mode decomposition (EEMD) and residual signal
}

\author{
Hafida Mahgoun ${ }^{\mathrm{a}}$, Rais Elhadi Bekka And Ahmed Felkaoui \\ Laboratoire de mécanique de précision appliquée (LMPA), Université Ferhat Abbas, Sétif 19000, Algérie
}

Received 11 November 2011, Accepted 8 December 2011

\begin{abstract}
This paper presents the application of new time frequency method, ensemble empirical mode decomposition (EEMD), in purpose to detect localized faults of damage at an early stage. EEMD is a self adaptive analysis method for non-linear and non-stationary signals and it was recently proposed by Huang and Wu to overcome the drawbacks of the traditional empirical mode decomposition (EMD). The vibration signal is usually noisy. To detect the fault at an early stage of its development, generally the residual signal is used. There exist different methods in literature to calculate the residual signal, in this paper we mention some of them and we propose a new method which is based on EEMD. The results given by the different methods are compared by using simulated and experimental signals.
\end{abstract}

Key words: Ensemble empirical mode decomposition (EEMD) / residual signal / gearbox fault diagnosis / fault detection / rotating machines

\section{Introduction}

Fault diagnosis of gearboxes has shown a great development in techniques based on the analysis of vibration signals [1-6], because vibration signals carry a great deal of information, which can be used to detect early faults in rotating machines. However, vibrations signals are influenced by vibration from many sources. Thus, the resulting signals are non stationary and nonlinear. To analyze such signals, time-frequency analysis has been applied to fault diagnosis of gearboxes in order to combine the advantages of both time and frequency domains.

Empirical Mode Decomposition (EMD) is a timefrequency analysis method, recently proposed by Huang et al. for the study of ocean waves $[7,8]$. The method has been developed and has been widely used [9-13]. In the field of fault diagnosis of rotating machines, the EMD method has also been widely applied for identification of faults $[5,14-20]$.

EMD is based on the local characteristic time scale of a signal and could decompose the complicated signal into a set of elementary signals called Intrinsic Mode Functions (IMFs). The IMFs represent the nature oscillation mode embedded in the signal and are determined by the signal itself. Thus, EMD is a self adaptive signal processing method and acts as a filter bank [21]. However, the original EMD has some drawbacks $[22,23]$. One of major

${ }^{a}$ Corresponding author: mahafida006@yahoo.fr drawbacks of the original EMD is the frequent appearance of mode mixing $[22,24]$, which is defined as a single intrinsic mode function (IMF), either consisting of signals of widely disparate scales, or a single of a similar scale residing in different IMF components. To overcome the problem of mode mixing in EMD, a noise assisted data analysis (NADA) method was proposed by Huang and $\mathrm{Wu}[25]$, it was called ensemble empirical mode decomposition EEMD. This method defines the true IMF components as the mean of an ensemble of trials, each consisting of the signal plus a white noise of finite amplitude. However, the measured signal is generally contaminated by noise that hides the information which is in direct relation with faults and may increase the amplitude of noise used by EEMD.

To alleviate these difficulties, in this work we use the EEMD method to calculate the residual signal (RS). The $\mathrm{RS}$ is obtained by removing some IMFs which represent the noise, the harmonics of the tooth meshing frequency and the regular signal.

By applying EEMD method in the calculation of the residual signal, we can decompose the signal at different levels and the change in the vibration signals caused by the localized fault is even more visible and the damage can be early identified.

The structure of the paper is as follows: Section 2 introduces the basic of EMD. Section 3 is dedicated to EEMD method. Section 4 presents the method and the procedure of the residual signal based on EEMD. 
In Section 5, we present examples of simulated signals to illustrate the behavior of the proposed algorithm. In Section 6 , the method is applied for gearbox faults diagnosis. In Section 7, a conclusion of this paper is given.

\section{Empirical mode decomposition (EMD) method}

\subsection{Review stage}

The empirical mode decomposition EMD is basically the output of an iterative algorithm $[7,8]$, it admits no analytical definition. The signal $x(t)$ can be decomposed as follows:

1. Identify all the local extrema, and then connect all the local maxima by a cubic spline line as the upper envelope.

2. Repeat the procedure for the local minima to produce the lower envelope. The upper and lower envelopes should cover all the data between them.

3. The mean of upper and lower envelopes value is designated as $m_{1}(t)$, and the difference between the signal $x(t)$ and $m_{1}(t)$ is $h_{1}(t)$

$$
h_{1}(t)=x(t)-m_{1}(t)
$$

4. If $h_{1}(t)$ is an IMF, then $h_{1}(t)$ is the first component of $x(t)$.

5. If $h 1(t)$ is not an IMF, $h_{1}(t)$ is treated as the original signal and repeat steps $(1-3)$; we got:

$$
h_{11}(t)=h_{1}(t)-m_{11}(t)
$$

in which, $m_{11}(t)$ is the mean of upper and lower envelopes value of $h_{1}(t)$.

6. After repeated sifting process $K$ times, $h_{1 k}(t)$ becomes an IMF, that is

$$
h_{1 k}(t)=h_{1(k-1)}(t)-m_{1 k}(t)
$$

then, it is designated $c_{1}(t)=h_{1 k}(t)$ as the first IMF component from the original data. $c_{1}(t)$ should contain the finest scale or the shortest period component of the signal.

7. Separate $c_{1}(t)$ from $x(t)$, we could get:

$$
r_{1}(t)=x(t)-c_{1}(t)
$$

8. $r_{1}(t)$ is treated as the original data, and repeat the above processes, the second IMF component $c_{2}(t)$ of $x(t)$ could be got.

9. Let us repeat the process as described above for $n$ times, then $n$-IMFs of signal $x(t)$ could be got. Then,

$$
\begin{gathered}
r_{1}(t)-c_{2}(t)=r_{2}(t) \\
\vdots \\
r_{n-1}(t)-c_{n}(t)=r_{n}(t)
\end{gathered}
$$

10. The decomposition process can be stopped when $r_{n}$ becomes a monotonic function, from which no more IMF can be extracted. By summing up (4) and (5), we finally obtain

$$
x(t)=\sum_{j=1}^{n} c_{j}(t)+r_{n}(t)
$$

the residue $r_{n}(t)$ is the mean trend of $x(t)$.

\subsection{The IMFs must satisfy the following definition}

- In the whole data set, the number of extrema and the number of zero-crossings must either be equal or differ at most by one.

- At any point, the mean value of the envelope defined by local maxima and the envelope defined by the local minima is zero.

\section{Ensemble empirical mode decomposition (EEMD) method}

The major drawback of the original EMD is the mode mixing $[24,25]$, which is the consequence of signal intermittence. The intermittence could cause the aliasing problem and makes the physical meaning of the IMF inclear. To overcome the mode mixing separation problem, a new noise-assisted data analysis (NADA) method is proposed. This method is named the Ensemble EMD (EEMD) [25], it defines the true IMF components as the mean of an ensemble of trials, each consisting of the signal plus a white noise of finite amplitude.

The proposed EEMD is defined as follows:

1. Add a white noise $y(t)$ to the original signal $x(t)$ to generate a new signal:

$$
x_{k}(t)=x(t)+\beta_{k} \cdot y(t)
$$

$\beta_{k}$ is a fraction of the standard deviation of the original signal $x(t)$.

2. Use the EMD to decompose the generated signals $x_{k}(t)$ into $n$ IMFs $c_{j k}(t), j=1, \ldots, n$, where $c_{j k}(t)$ is the $j$ th IMF of the $k$ th trial.

3. Repeat steps (1) and (2) $K$ times with different white noise series each time to obtain an ensemble of IMFs: $c_{j k}(t), k=1, \ldots, K$.

4. Determine the ensemble mean of the $K$ trials for each IMF as the final result:

$$
c_{j}(t)=\lim _{K \rightarrow \infty} \frac{1}{K} \sum_{k=1}^{K} c_{j k}(t), j=1, \ldots, n
$$

To remove the influence of some indesired components as the noise and to show clearly the signal components generated by the crack damage, we propose to use the residual signal. 
There exist different methods to calculate the residual signal, in this work, first we give a preview on some methods that already exist and then we propose a new method based on the ensemble empirical mode decomposition.

\section{Residual signal}

The idea of residual signal was first proposed by Stewart [26] in 1977. The purpose of the residual signal is to eliminate the components which occur at the fundamental and harmonics of the tooth meshing frequency from the time average. The time domain averaging is based on obtaining a signal describing one revolution of the considered gear by averaging the vibration signal over a number of revolution. It used to eliminate all the components not synchronized to the shaft including noise.

Then the objective of getting the residual signal is to remove the influence of the noise and the regular vibration components and show the signal components generated by crack damage.

For healthy gears, the gear meshing frequency, its harmonics, the shaft rotation frequencies and their harmonics dominate the vibration spectrum.

When a local gear fault such as a crack in a tooth is present, the vibration signal in a complete revolution will be modified by the effects of a short duration impact impulse. In order to detect the fault features in the vibration signal, the regular components need to be removed; the rest is called the residual signal, which is supposed to be more sensitive to crack growth.

In this study, we will examine three different methods of generating residual signals.

(a) The first method is based on removing the mesh harmonics from the time synchronous average signal (TSA).

$$
x_{\mathrm{res}}(t)=x_{\mathrm{TSA}}(t)-\frac{1}{z} \sum_{i=0}^{z-1} x\left(t+i T_{e}\right)
$$

where, $x_{\text {res }}$ is the residual signal, $x$ is the vibration signal, $Z$ is the number of teeth of the gear, $T_{e}$ is the mesh period and $x_{\mathrm{TSA}}$ is the time synchronous average signal that is given by the next equation.

$$
x_{\mathrm{TSA}}(t)=\frac{1}{N} \sum_{i=0}^{N-1} x(t+i T)
$$

where, $T$ is one rotation period and $N$ is the number of periods.

(b) The second method is based on the signal when there is no crack that can be considered to be the regular signal, and the residual signal is obtained by removing the regular signal from the cracked vibration signals [27]. In our work, we consider that the signal of the second day correspond to the signal without crack.

(c) The third method which is proposed in this paper, is based on the EEMD method. The residual signal is obtained by removing some IMFs which represent the harmonics of the tooth meshing frequency, the noise and the regular signal.

In this section, we present a brief description of the algorithm used to get the residual signal from EEMD method. Figure 1 shows the block diagram of the method, which comprises the following steps:

1. The EEMD algorithm is used to decompose the signal into intrinsic mode functions, $I M F_{1} \ldots I M F_{n}$, where $n$ is the number of IMFs.

2. We calculate the time synchronous average of each IMF by applying the next equation:

$$
I M F_{\mathrm{TSA}}(t)=\frac{1}{N} \sum_{i=0}^{N-1} I M F(t+i T)
$$

3. We calculate the residual IMF, by applying the next equation:

$$
I M F_{\mathrm{res}}(t)=I M F_{\mathrm{TSA}}(t)-\frac{1}{Z} \sum_{i=0}^{Z-1} I M F\left(t+i T_{e}\right)
$$

4. Kurtosis is used in the measurement of impulsiveness, for this purpose we calculate the Kurtosis of each residual IMF. In this stage, the residual IMFs that have a Kurtosis less or equal to 3 were eliminated.

5. We calculate the residual signal by adding together all the residual IMFs that have a Kurtosis superior than 3 .

$$
x_{\mathrm{res}}(t)=\sum_{j=1}^{n} I M F_{\mathrm{res}} j(t)
$$

6. Calculate the Kurtosis of the residual signal to check if there are any impulses in the residual signal.

\section{Simulation}

This section provides validation of the efficiency of the method used in this paper to get the residual signal.

\subsection{Example 1}

Let us consider a vibration signal $x(t)$ consisting of two components $x_{1}(t)$ and $x_{2}(t)$ (Fig. 2), where $x_{1}(t)$ represents the periodic components in the vibration signal, given by:

$$
x_{1}(t)=\sin (2 \pi 50 t)+\sin (2 \pi 120 t) \quad 0 \leq t \leq 0.06 \mathrm{~s}
$$

and the term $x_{2}(t)$ represents the transient component produced by the fault, given by:

$$
x_{2}(t)=A 1(t) \cdot A 2(t) \quad 0 \quad \leq t \leq 0.06 \mathrm{~s}
$$




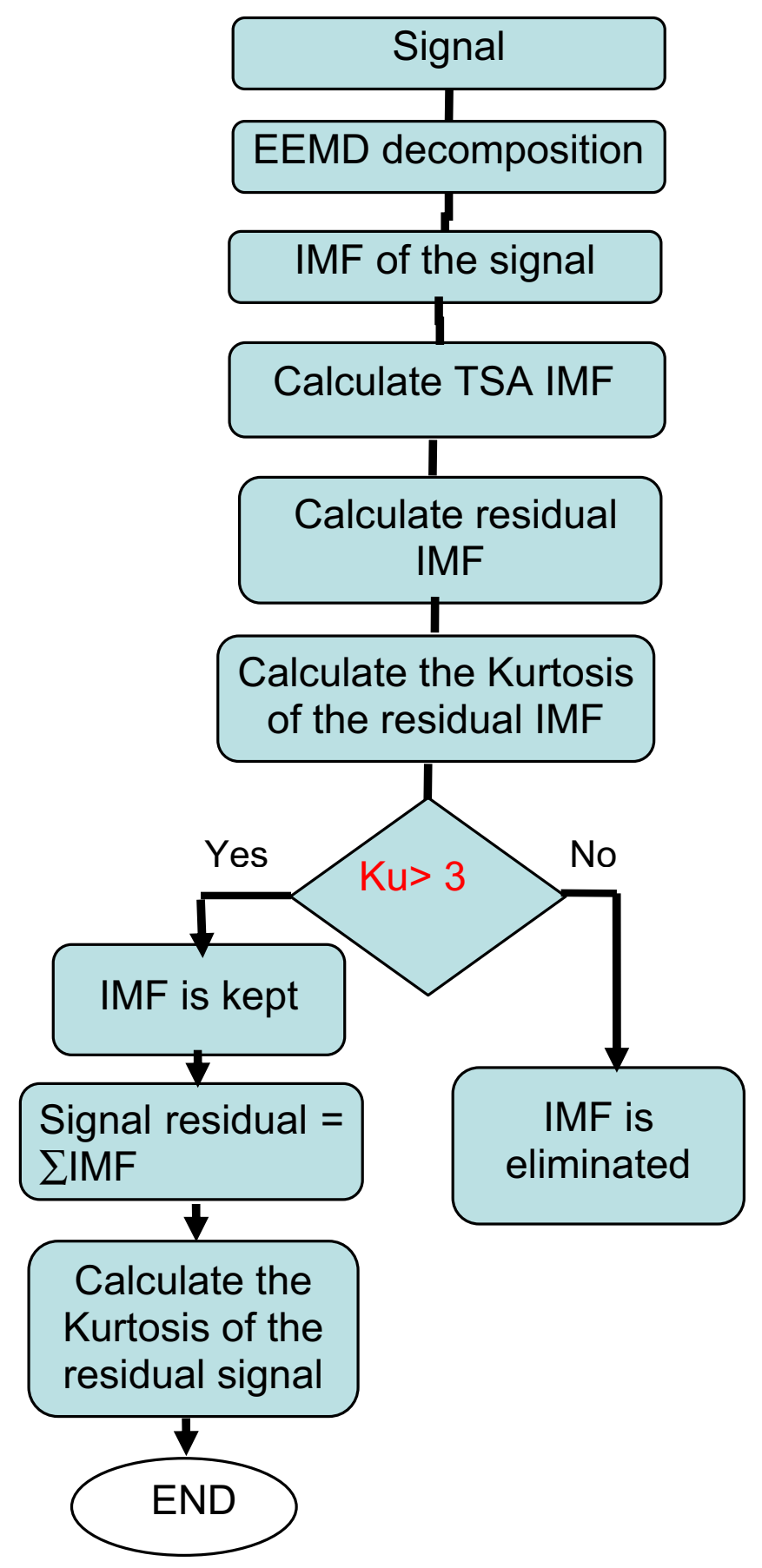

Fig. 1. Diagram of the EEMD residual signal.

where, $A 1(t)=0.05 \sin (2 \pi 2000 t)$ and

$A 2(t)=e^{(t-0.005)^{2} / 10^{-6}}+e^{(t-0.025)^{2} / 10^{-6}}+e^{(t-0.045)^{2} / 10^{-6}}$

The Kurtosis value of $x(t)$ is 2.28 , this value does not indicate the presence of any impulse. The FFT spectrum of the signal is given in Figure 3. It shows only the frequencies which represent the periodic part of the signal.

By using EMD, the signal $x(t)$ is decomposed and the results shown in Figure 4 are obtained. It is obvious that
Table 1. Kurtosis values of each IMF for the normal gear.

\begin{tabular}{ccccc}
\hline IMF & Signal & IMF1 & IMF2 & IMF3 \\
\hline Kurtosis & 2.501 & 2.13 & 2.03 & 1.53 \\
\hline
\end{tabular}

the IMFs obtained by EMD are distorted seriously and the mode mixing is occurring between IMFs.

The simulation signal in Figure 2 is decomposed again using EEMD. The decomposition result is shown in Figure 5. From this figure, it is seen that the components included in the signal are decomposed into separate IMFs perfectly. The first IMF corresponds to the transient component; the second IMF and the third IMF indicate the periodic components, and the last IMF corresponds to the residue. Then, the different components embedded in the signal can be extracted accurately using the EEMD method.

We can see also that the Kurtosis of the first IMF $(\mathrm{Ku}=16.88)$ is greater than 3 , and the Kurtosis of the other IMFs is less than 3, which allows us to calculate the residual signal by taking only the first IMF $(\mathrm{Ku}>3)$.

\subsection{Example 2}

Consider a normal pair of gears meshing under a constant load and speed, and which have different number of teeth. Suppose that the teeth on each gear are identical and are free of eccentricity. The vibration signal generated by the gears can be represented as [28, 29]:

$$
x_{N G}(t)=\sum_{m=0}^{M} X_{m} \cos \left(2 \pi m Z f_{r} t+\varphi_{m}\right)
$$

where, $M$ is the number of tooth-meshing harmonics, $X_{m}$ and $\varphi_{m}$ are, respectively, the amplitude and the phase of the $m$ th meshing harmonic, $Z$ is the number of gear teeth, $t$ is time, $f_{r}$ is the shaft rotation frequency, and $f_{m}=$ $Z . f_{r}$ is the meshing frequency. Equation (17) indicates that the vibration signal acquired from a normal gearbox generally exhibits predominant frequency components at the meshing frequency and its harmonics. Figure 6 shows the EEMD decomposition of a simulated vibration signal from a normal gearbox that have the same features that the experimental test bench (a gear with 21 teeth and rotational frequency is $16.67 \mathrm{~Hz}$, the meshing frequency is $333 \mathrm{~Hz}$, the number of harmonics is $M=3$, the sampling frequency is $f e=20000 \mathrm{~Hz}, N=4096$ points, and the number of sidebands $M 1=5$ ). Table 1 gives the Kurtosis values calculated for each IMF.

Suppose that the gearbox has a tooth fault then the generated signal is modulated by the gear rotating frequency and contains amplitude and phase modulations that are periodic with the rotating frequency of the faulty gear.

The modulation of the meshing frequency generates sidebands, which are frequency components equally spaced around the carrier frequency. Changes in vibration generated by a faulty gear tooth can be represented 


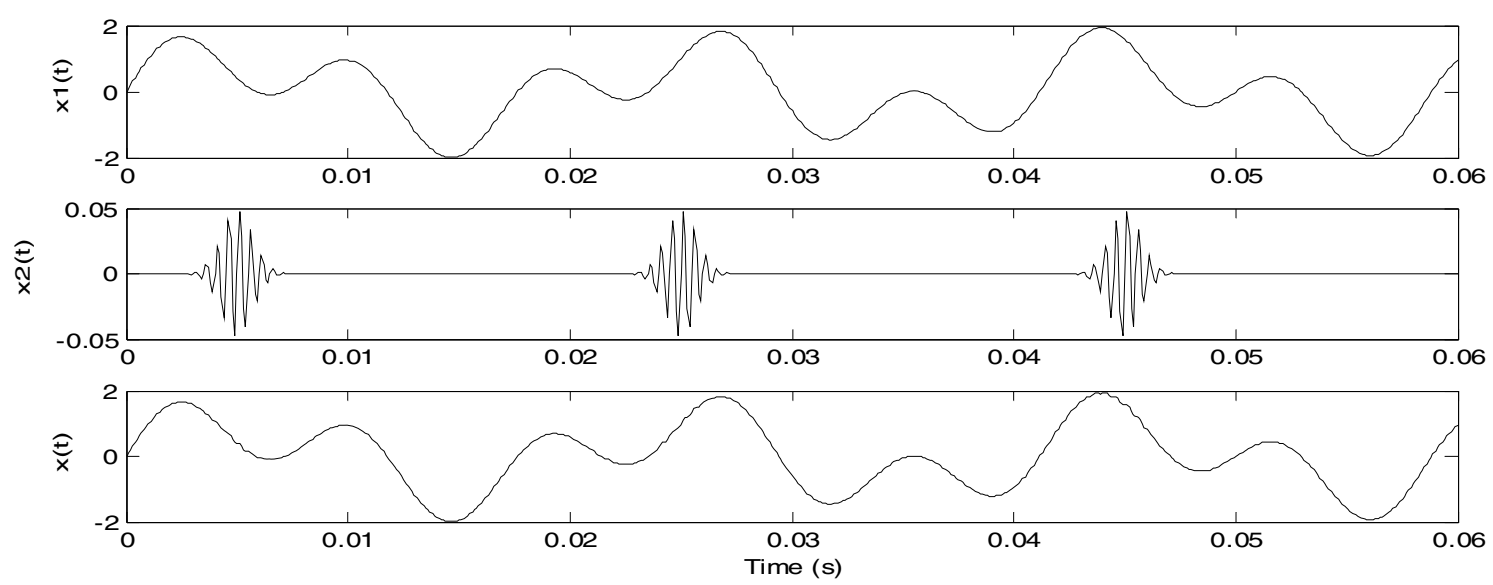

Fig. 2. The two components and the simulation signal.

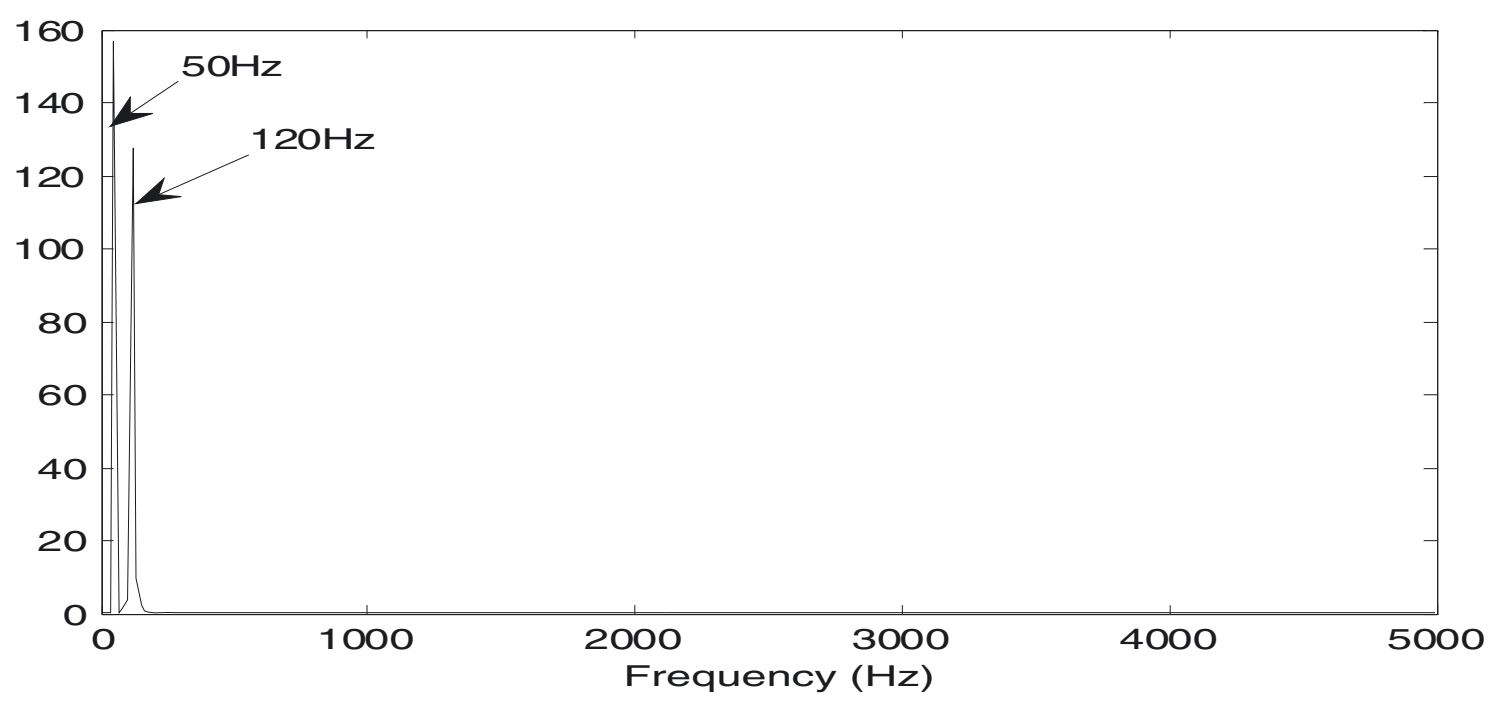

Fig. 3. FFT spectrum of the simulation signal.

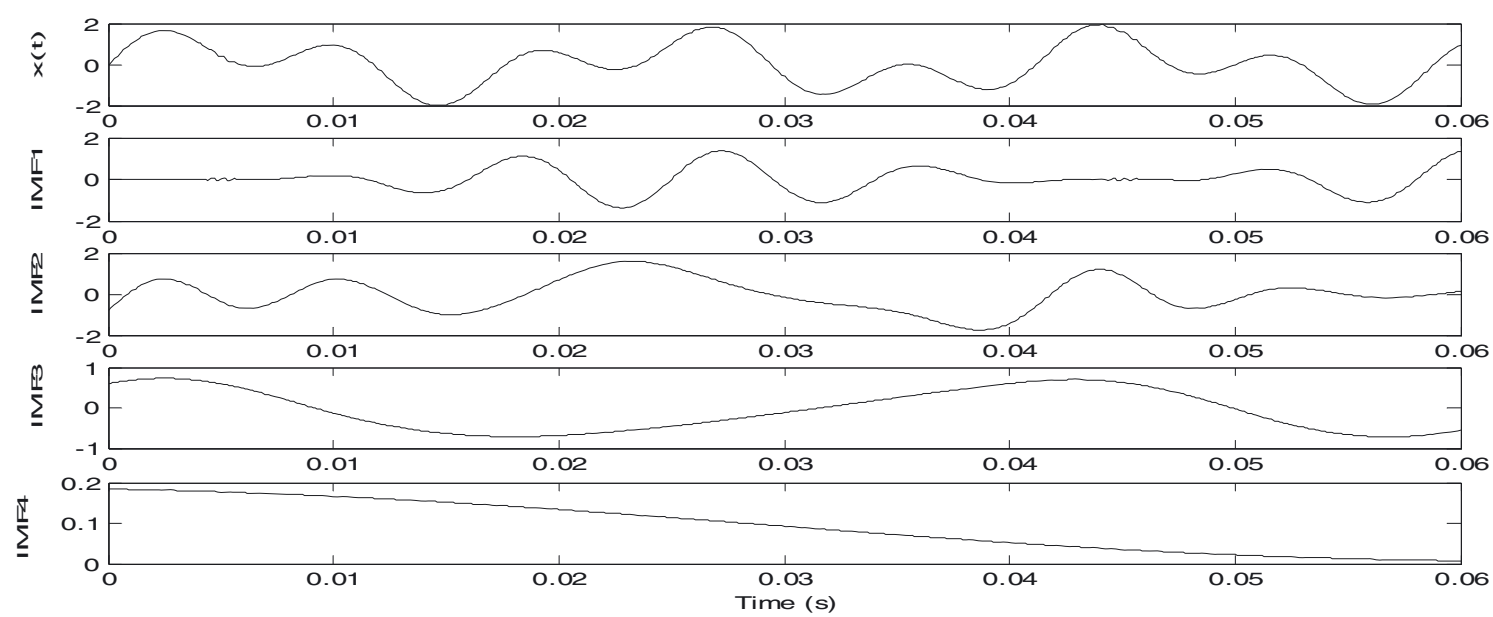

Fig. 4. The decomposition result with EMD. 

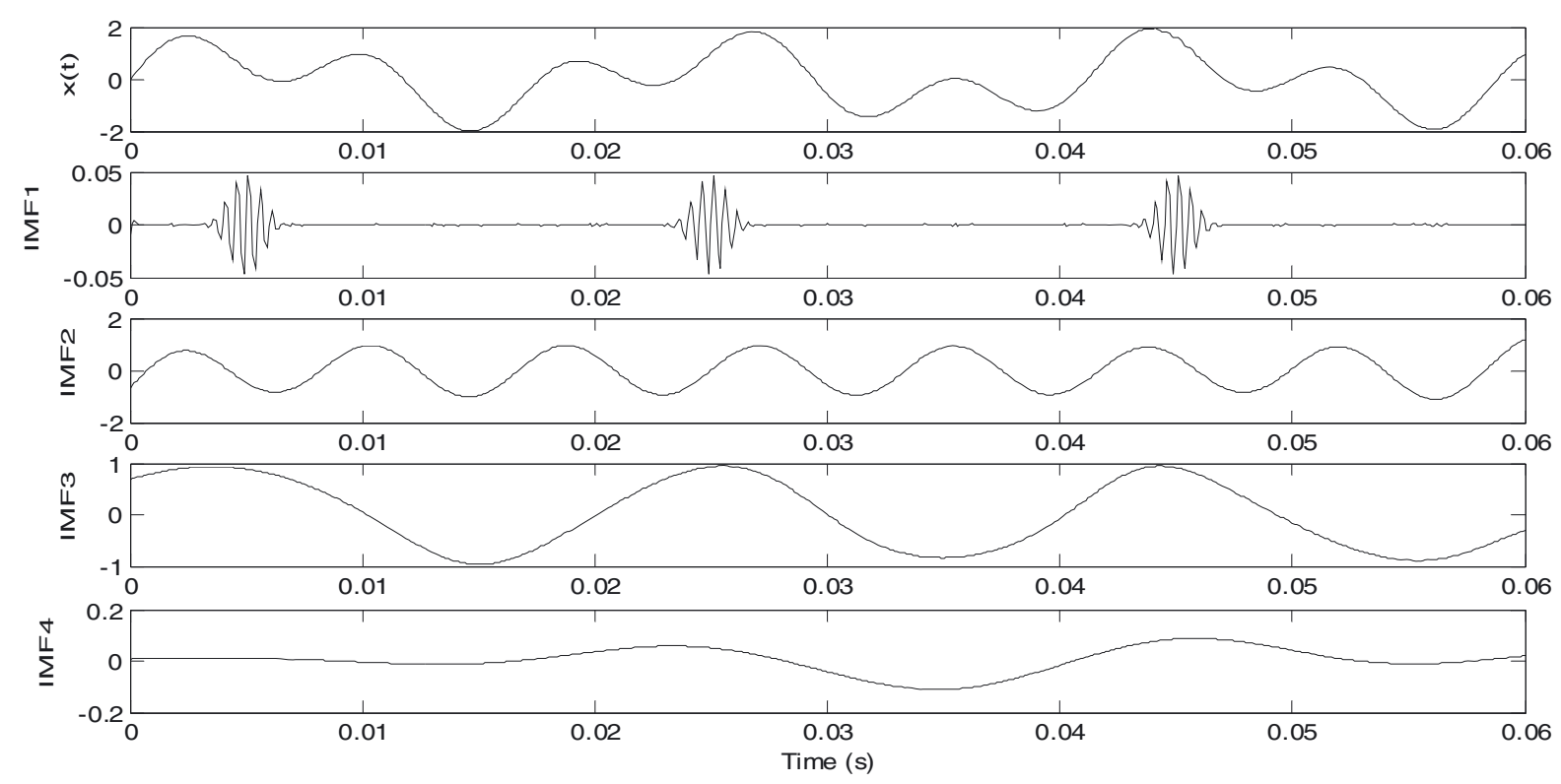

Fig. 5. The decomposition result with EEMD.
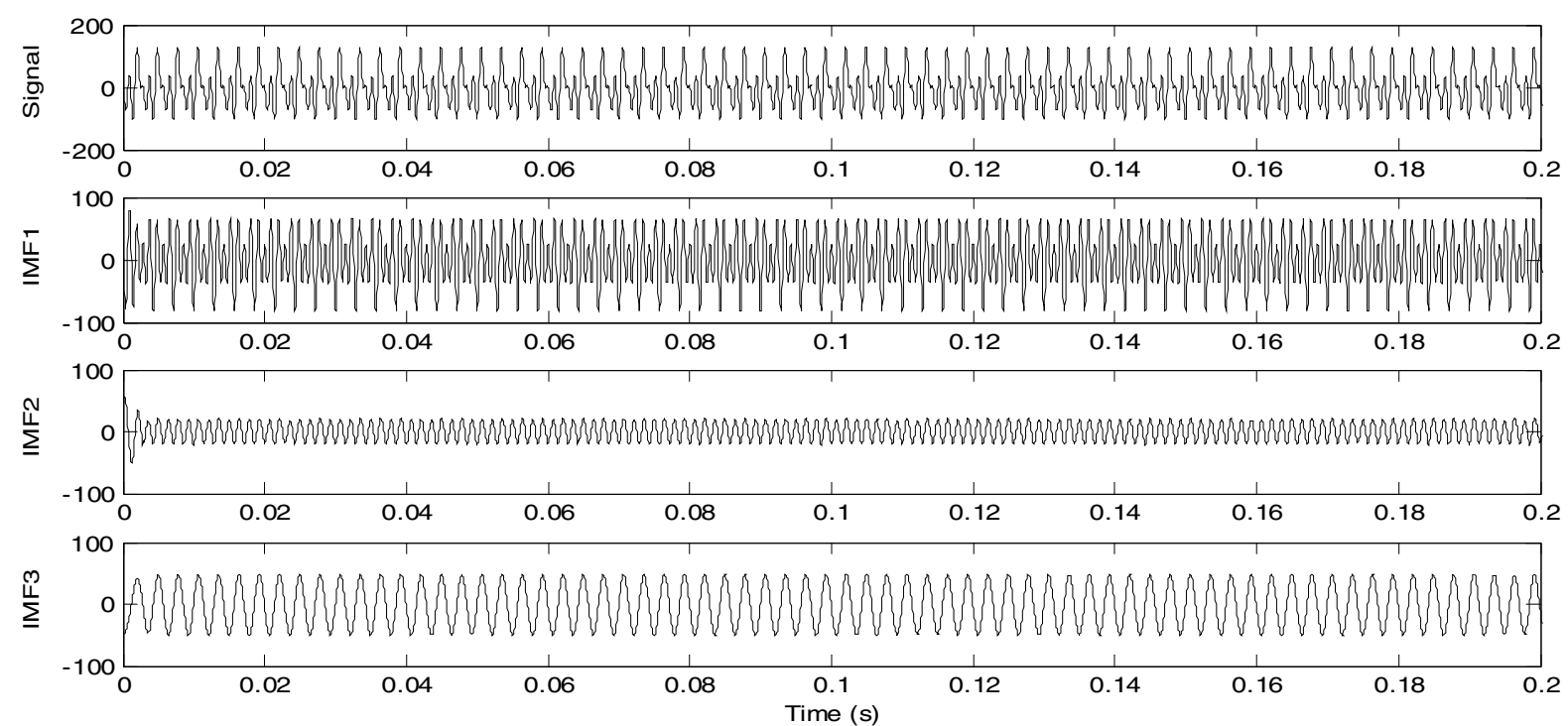

Fig. 6. The decomposition results of a simulated vibration signal from a normal gearbox.

by the following amplitude and phase-modulating functions, $a_{m}(t)$ and $b_{m}(t)$, respectively:

$$
\begin{aligned}
& a_{m}(t)=\sum_{n=0}^{M 1} A_{m n} \cos \left(2 \pi n f_{r} t+\alpha_{m n}\right) \\
& b_{m}(t)=\sum_{n=0}^{M 1} B_{m n} \cos \left(2 \pi n f_{r} t+\beta_{m n}\right)
\end{aligned}
$$

where $M_{1}$ is the number of sidebands around toothmeshing harmonics, $A_{m n}$ and $B_{m n}$ are amplitudes at the nth sidebands of amplitude and phase-modulating signals, respectively, around the $m$ th meshing harmonic, $\alpha_{m n}$ and $\beta_{m n}$ are phases at the $n$th sideband of amplitude and phase-modulating signals, respectively, around the $m$ th meshing harmonic. The combined modulated vibration signal $x(t)$, produced by a pair of meshing gears with a tooth fault, is given by

$x(t)=\sum_{m=0}^{M} X_{m}\left(1+a_{m}(t)\right) \cdot \cos \left(2 \pi m Z f_{r} t+\varphi_{m}+b_{m}(t)\right)$

Figure 7 shows the time domain and the frequency domain of a simulated vibration signal from a faulty gearbox. The time domain presents a signal in the form of impacts. In the same time the frequency domain is consists of the meshing frequency and its harmonics and sidebands around them, in the same time, we can see that the spread of the peaks is larger than the normal condition.

Figure 8 shows the EEMD decomposition of the simulated vibration signal from a faulty gear tooth, we see that 

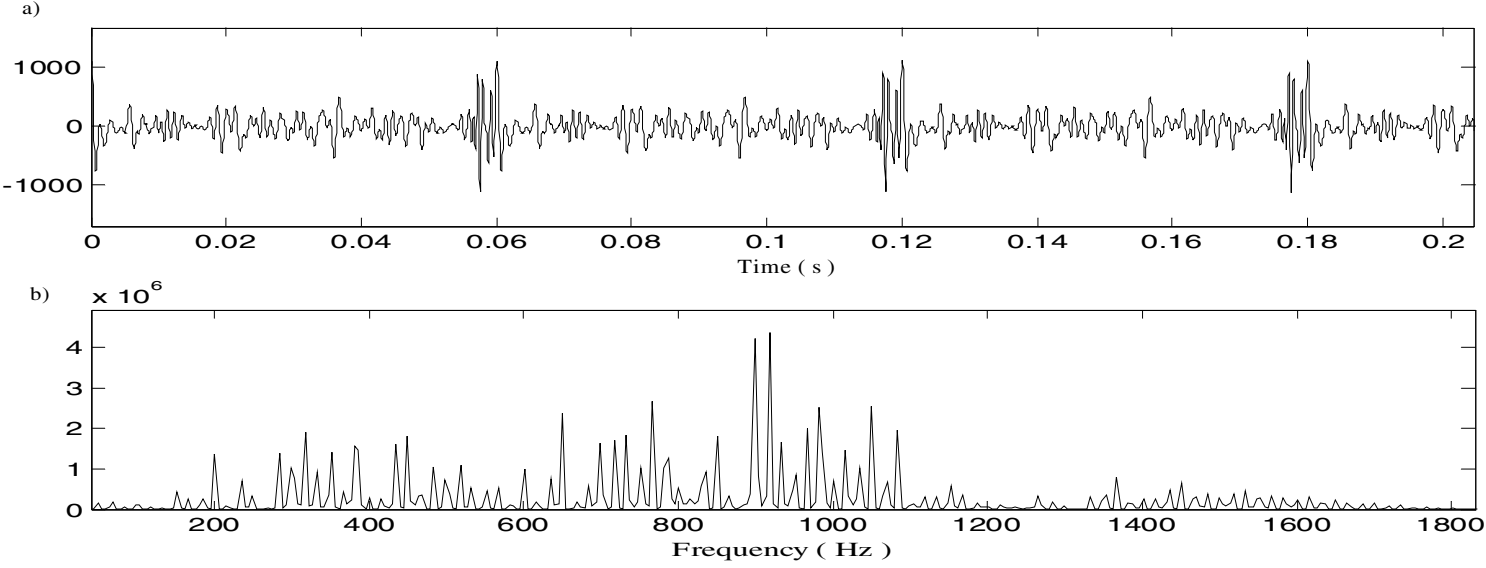

Fig. 7. Simulated vibration signal from a faulty gear tooth, (a) time domain, (b) frequency domain.
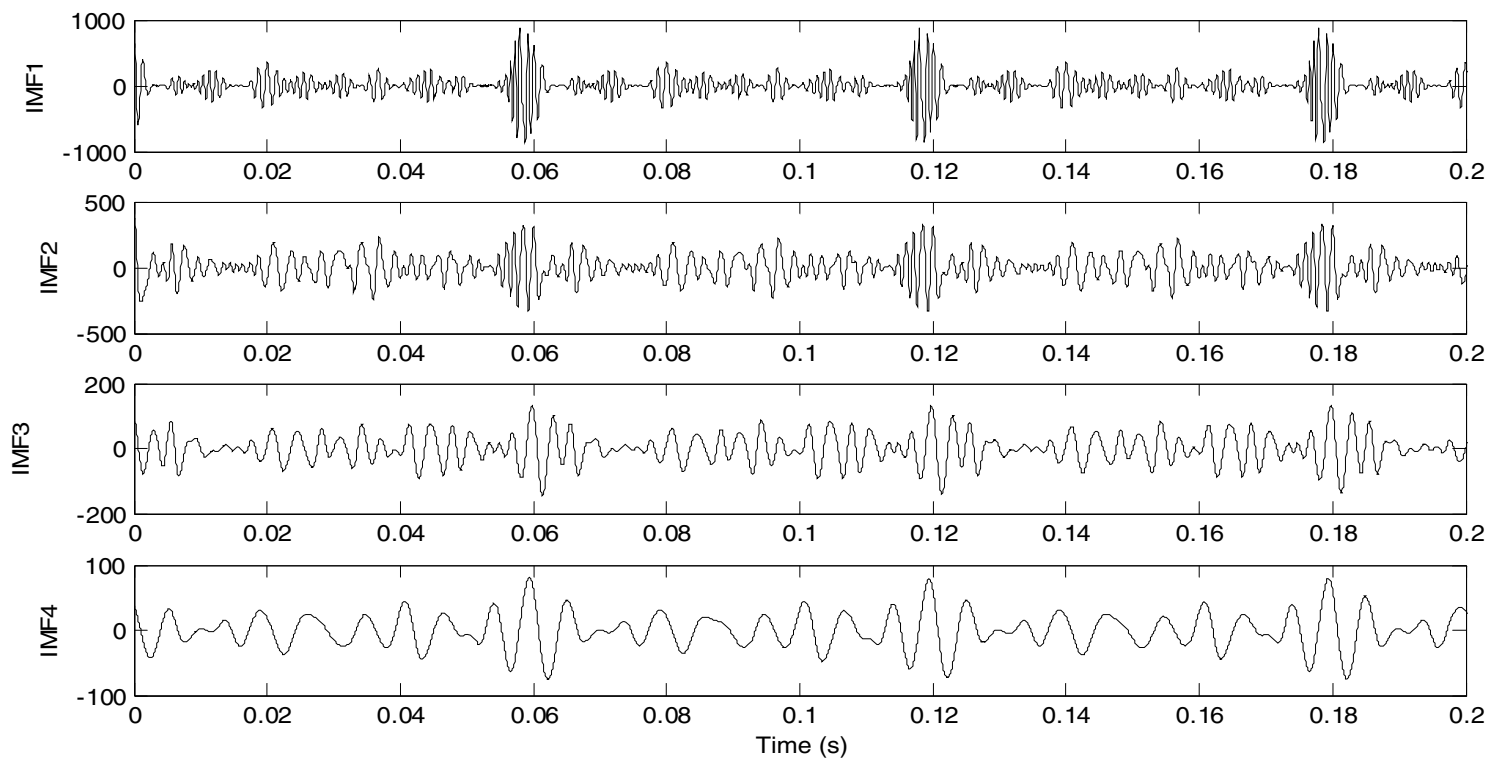

Fig. 8. The decomposition results of a simulated vibration signal from a faulty gear tooth.

the first IMFs are in the form of impacts. The Kurtosis values of each IMF of the cracked signal are given in Table 2, the IMFs from 8 to 11 are characterized by a very low amplitude, for this reason, we have added them to the residue.

To explain the idea of the application of the residual signal, we have applied the three methods to calculate the residual signal, and then we have calculated the Kurtosis of the residual signal for normal and faulty gear. In the same time, we have calculated the Kurtosis of the residual IMFs (Tab. 2) given by the use of Equation (12). By applying the three methods, we have got the results summarized in Table 3 .

These results show that all the three methods give good results but, the third method based on EEMD permits to decompose the signal at different levels and calculate the Kurtosis of different bands of frequency, which allow us to follow the advancement of default in high and low frequency.
Table 2. Kurtosis values for the raw and the residual IMFs of the faulty gear.

\begin{tabular}{ccc}
\hline \multirow{2}{*}{ IMFs } & \multicolumn{2}{c}{ Kurtosis } \\
\cline { 2 - 3 } & Raw signal & Residual signal \\
\hline IMF1 & 9.67 & 21.30 \\
IMF2 & 3.62 & 13.64 \\
IMF3 & 3.19 & 16.62 \\
IMF4 & 3.34 & 18,14 \\
IMF5 & 2.27 & 10.13 \\
IMF6 & 1.99 & 7.67 \\
IMF7 & 2.01 & 5.09 \\
IMF8 & 2.89 & 2.34 \\
IMF9 & 2.97 & 2.18 \\
IMF10 & 2.68 & 2.42 \\
IMF11 & 1.66 & 1.50 \\
\hline
\end{tabular}

\section{Application to experimental data}

The vibration signals used in this work were carried out at CETIM, France $[3,17]$. 
Table 3. The Kurtosis values of residual signals.

\begin{tabular}{cccc}
\hline $\begin{array}{c}\text { Residual } \\
\text { signal }\end{array}$ & $\begin{array}{c}\mathrm{Ku} \\
\text { 1st method }\end{array}$ & $\begin{array}{c}\mathrm{Ku} \\
\text { 2nd method }\end{array}$ & $\begin{array}{c}\mathrm{Ku} \\
\text { 3rd method }\end{array}$ \\
\hline Faulty gear & 21.91 & 6.84 & 21.90 \\
\hline
\end{tabular}

The system under test is composed of a pair of spur gears which have a number of teeth respectively $Z_{1}=$ 20 teeth and $Z_{2}=21$ teeth, the 20 teeth gear has a rotation speed of $1000 \mathrm{rpm}$, the experiment was carried out for 13 days length and the measurements were collected every $24 \mathrm{~h}$, on the last day the fault was in an advanced stage close to the breakage of the tooth.

The rotating speed of the input shaft is: $f r_{1}=$ $16.67 \mathrm{~Hz}$ and the rotating speed of the output shaft is $f r_{2}=15.87 \mathrm{~Hz}$, the meshing frequency is $f_{e}=Z_{1} \cdot f_{r 1}=$ $Z_{2} . f_{r 2}$, then $f_{e}=333 \mathrm{~Hz}$.

This test bed has been studied by other researchers $[3$, $17,30,31]$. They have used new signal processing techniques.

In the first paper, Capdessus et al. [3] have used the cyclostationarity analysis. They have used the synchronised averaging (first-order cyclostationarity) in order to eliminate all the components not synchronised to the shaft including random noise. The fault on the gear was detected from the ninth day.

In the second paper, Parey et al. [17] have used for early detection of this localized tooth defect, EMD and a statistical analysis based on Kurtosis. First, they have decomposed the signal in IMFs, and then they have calculated directly the Kurtosis of these IMFs. They have not used any technique to remove the noise. So, the fault was detected from the tenth day, and then, the elimination of noise gives best results.

In third paper Badaoui et al. [30] have used the power cepstrum and a new indicator which is not affected by the signal amplitude and the signal-to-noise ratio. And from the evolution of this indicator they conclude that the pinion was developing a defect from the eighth day.

In the last work [31], the author has shown that the spectral correlation could detect the default at an early stage (sixth day).

Figure 9 shows the acceleration signals in time domain, the signals correspond to five days of the experiment (2nd day, 6th day, 9th day, 11 th day and 13 th day). The time trend of the vibration signals corresponding to 2 nd day, 6 th day, 9 th day, 11 th day indicate that it is not possible to detect the fault of the gear only by looking at the time trend plot, since the firsts impulses due to appearance of faults are masked by the noise. For the vibration signals corresponding to the last day 13th, we can see that the fault is characterized by periodical impulses caused by cracked teeth. Then the fault is in advanced stage.

In this study, first we have calculated the Kurtosis values for acceleration signals from day 2 to 13 (Tab. 4), then each signal was decomposed by the EEMD method, Figures 10-12 display IMFs given by EEMD which correspond to the 2 nd day, the 9 th day and the last day. We
Table 4. Kurtosis values for raw and residual signals.

\begin{tabular}{ccc}
\hline Days & $\begin{array}{c}\text { Ku of the } \\
\text { raw signal }\end{array}$ & $\begin{array}{c}\text { Ku of the residual } \\
\text { signal (3rd method) }\end{array}$ \\
\hline 2 & 2.734 & 3.14 \\
3 & 2.686 & 3.35 \\
4 & 2.963 & 3.99 \\
5 & 2.776 & 5.27 \\
6 & 3.084 & 4.19 \\
7 & 2.989 & 4.41 \\
8 & 2.972 & 4.30 \\
9 & 2.870 & 4.66 \\
10 & 3.169 & 4.27 \\
11 & 3.118 & 4.33 \\
12 & 12.64 & 13.64 \\
13 & 13.44 & 15.77 \\
\hline
\end{tabular}

Table 5. The expert report

\begin{tabular}{cc}
\hline Days & Observations \\
\hline 2 & First day of acquisition, no anomaly \\
3 & No anomaly \\
4 & $/ / / / /$ \\
5 & $/ / / /$ \\
6 & Chipping tooth $1 / 2$ \\
7 & No evolution \\
8 & Tooth $1 / 2$ no evolution \\
& $15 / 16$ beginning of chipping \\
9 & Tooth $1 / / / /$ \\
10 & Evolution of chipping tooth $15 / 16$ \\
12 & $/ / /$ \\
13 & Chipping over all the width of tooth $15 / 16$ \\
\hline
\end{tabular}

have obtained 12 IMFs for each signal, we have presented only nine IMFs, the last IMFs are added to the residue. These figures show that the first IMF indicates the highest frequencies contained in the signal and we have remarked that rotation frequency is presented by IMF 8 or IMF 9 .

After that, we have calculated the residual IMFs from Equation (12). By subtracting the residual IMFs that the Kurtosis is less than 3, we have obtained the residual signal. Finally, we have calculated the Kurtosis values for the residual signals (Tab. 4).

The variation of the Kurtosis values indicates that the fault was started from the fifth day. Also we can distinguish clearly three different stages (Fig. 13), the first one starts from day 2 to day 4 and it corresponds to the no anomaly, the second period starts from day 5 to day 11 this stage corresponds to the appearence of the fault which is a chipping tooth and finaly, the last stage where the default is in advanced stage and corresponds to the evolution of chipping tooth. These results are justified by the expert report given in Table 5 .

\section{Conclusion}

The EEMD decomposition provides a powerful tool for non-stationary and non-linear signal analysis. The results presented in this study demonstrate that the combination 


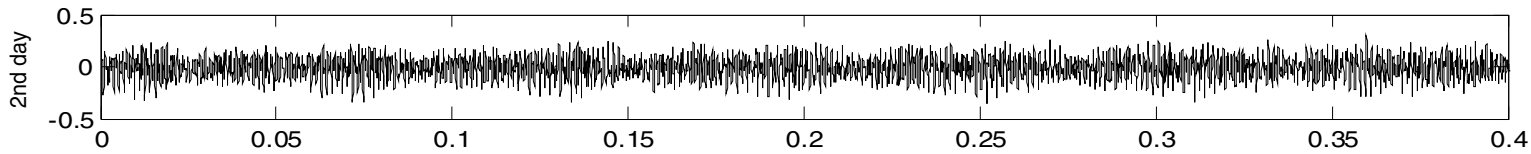

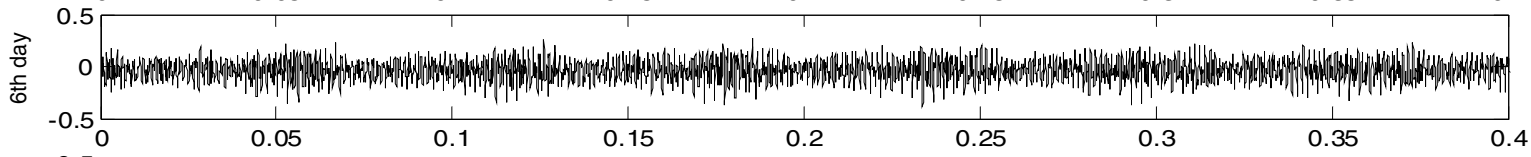
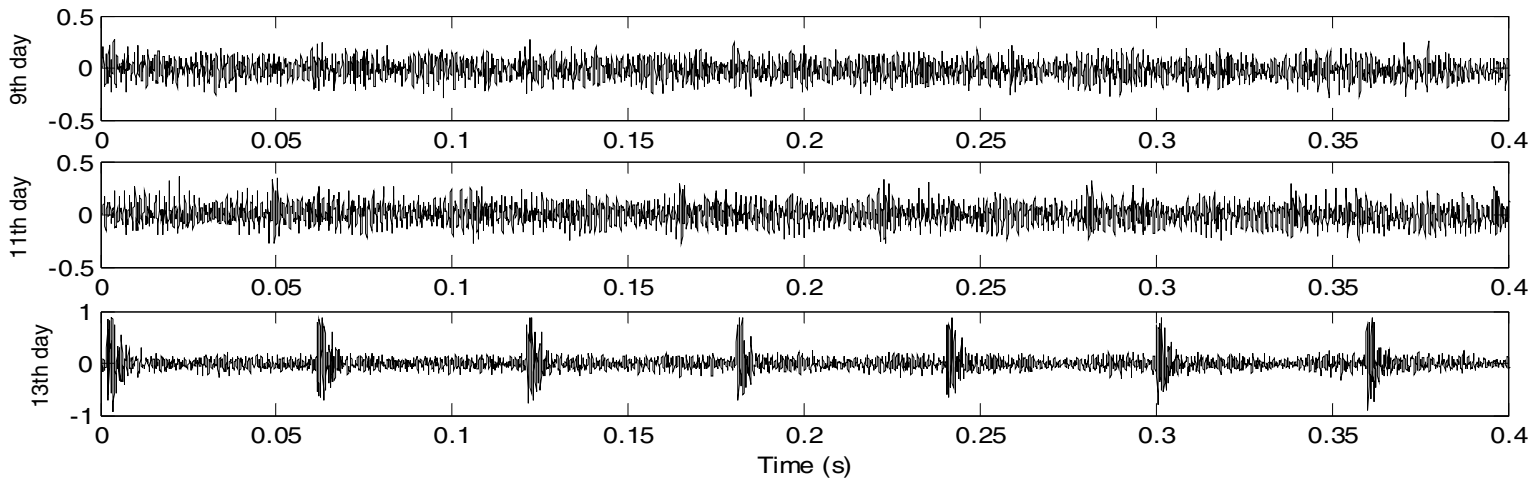

Fig. 9. Analyzed vibration signals of the 2 nd day, 6 th day, 9 th day, 11 th day and 13 th day.
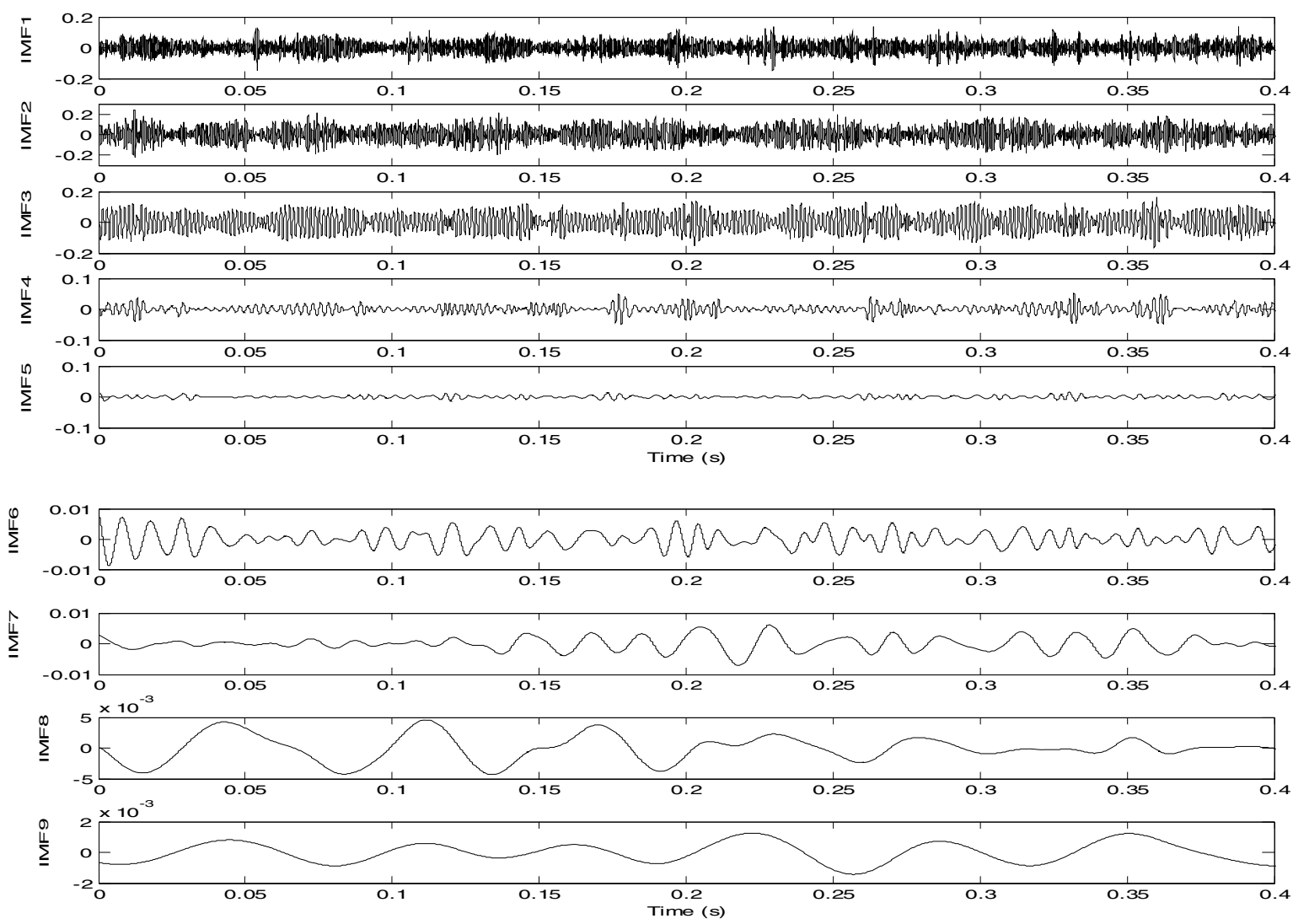

Fig. 10. EEMD of the signals of the 2nd day. 


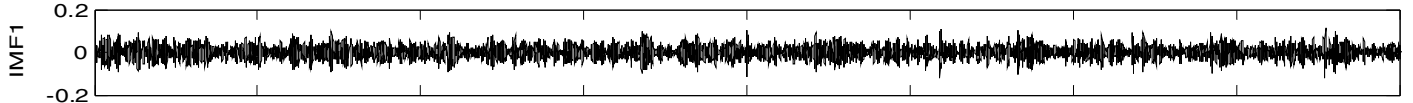

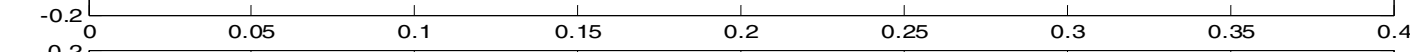

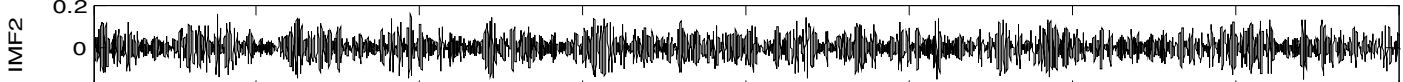

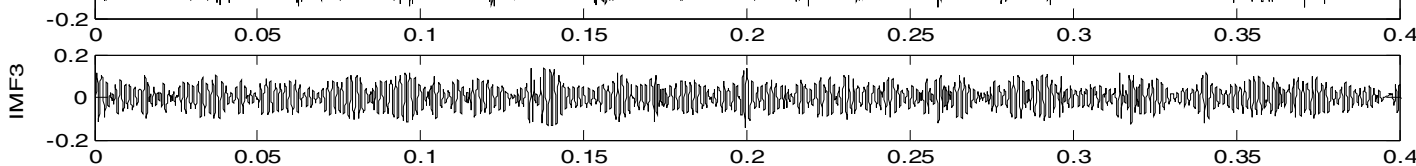

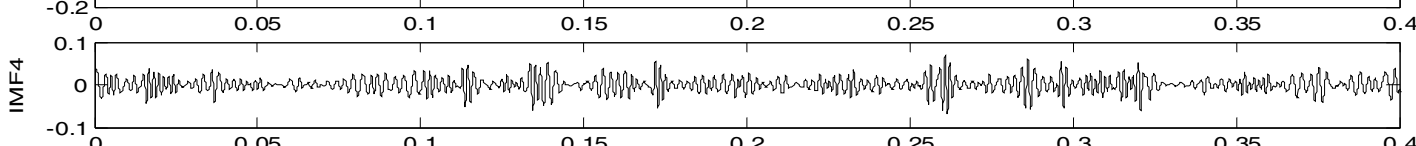

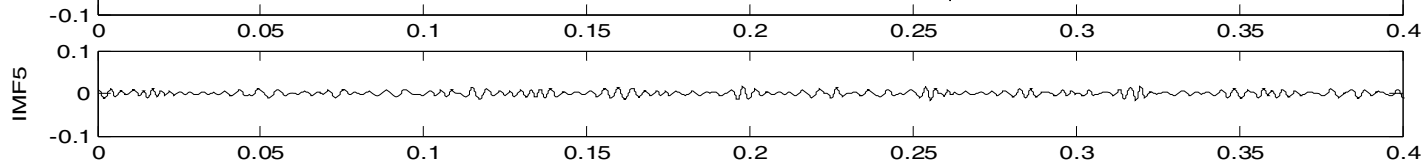

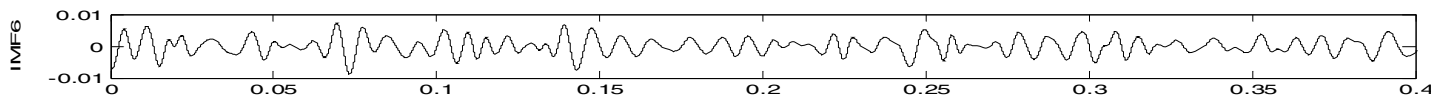

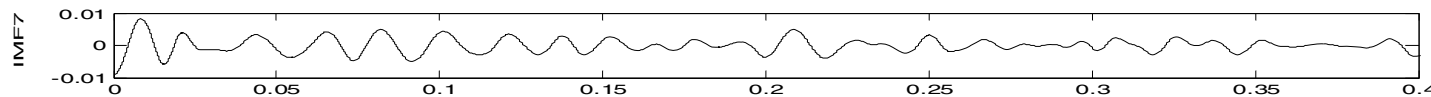
0.05
$\underline{5}$

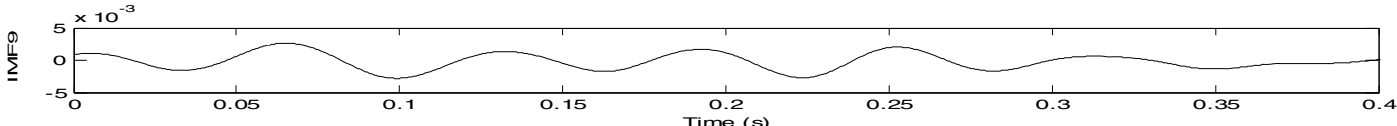

Fig. 11. EEMD of the signals of the 9th day.
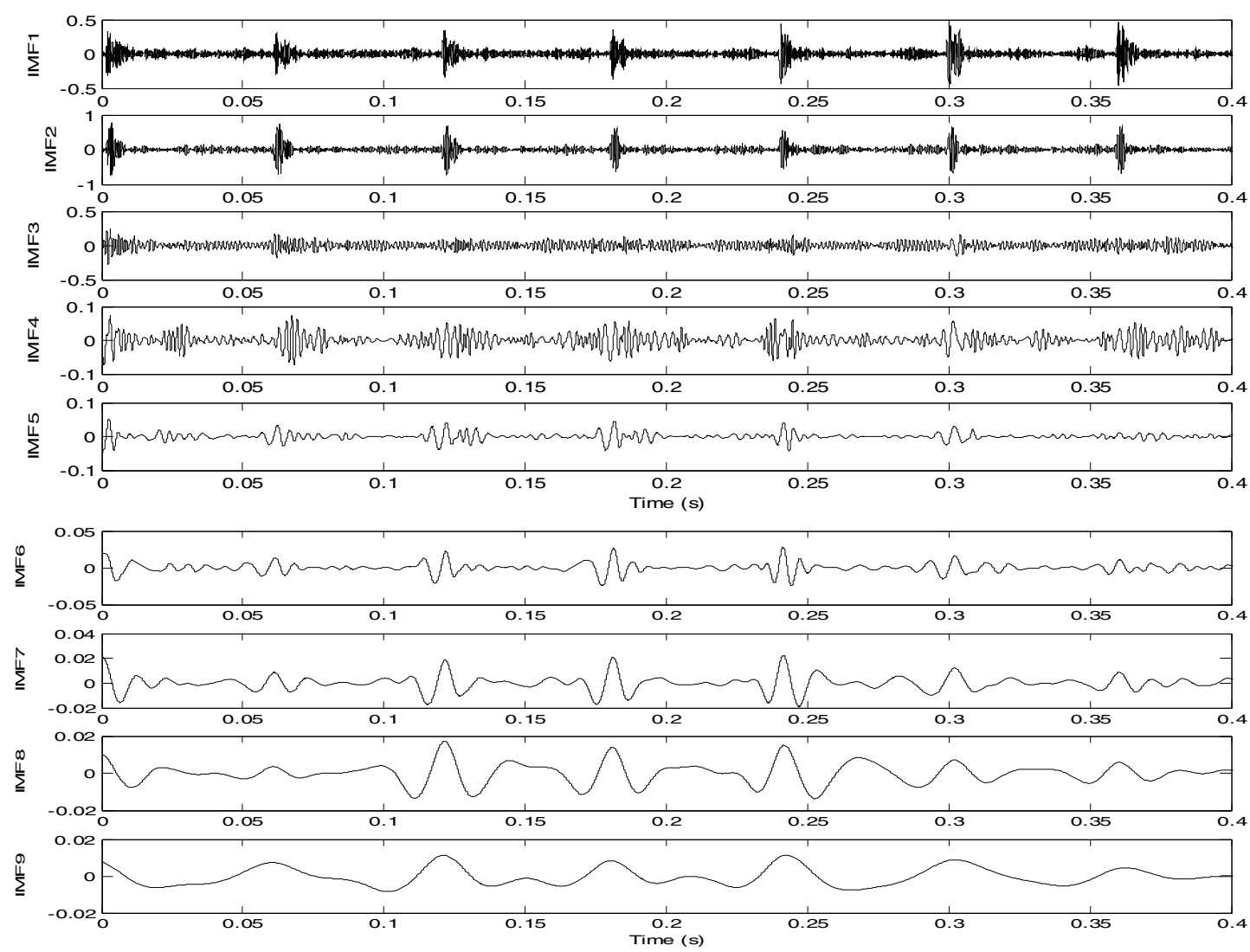

Fig. 12. EEMD of the signals of the 13th day. 


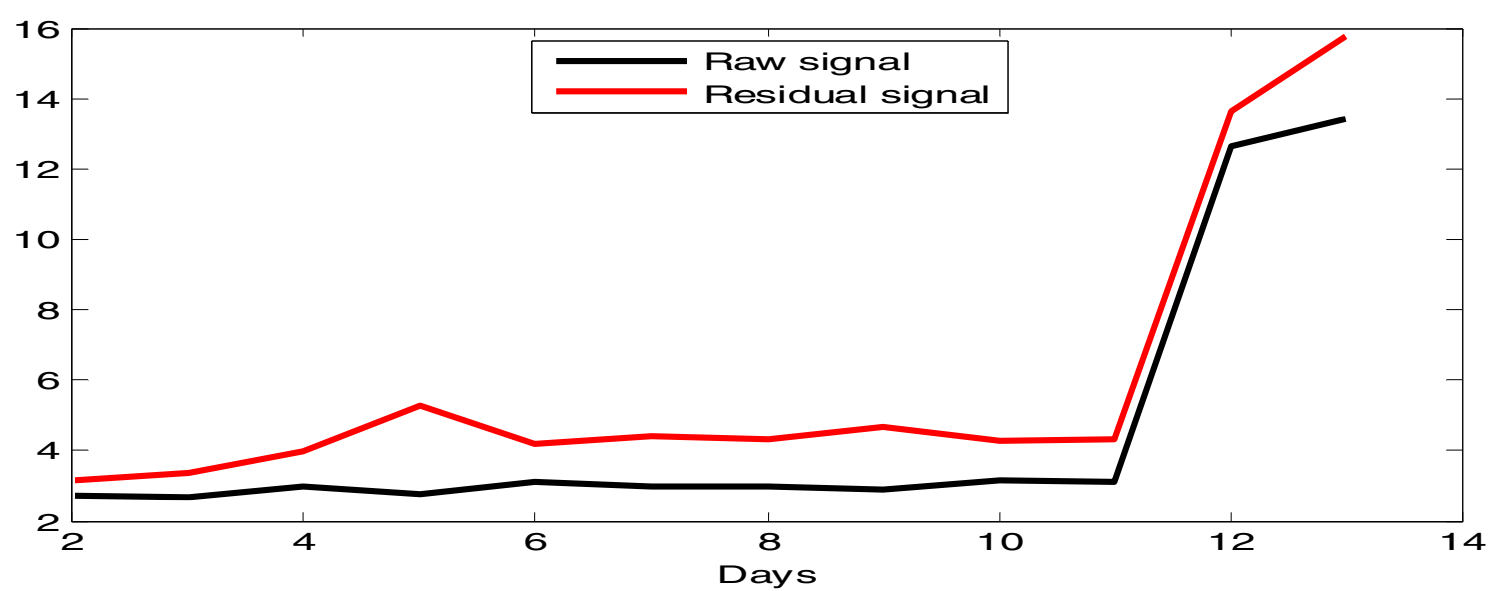

Fig. 13. Kurtosis values variation.

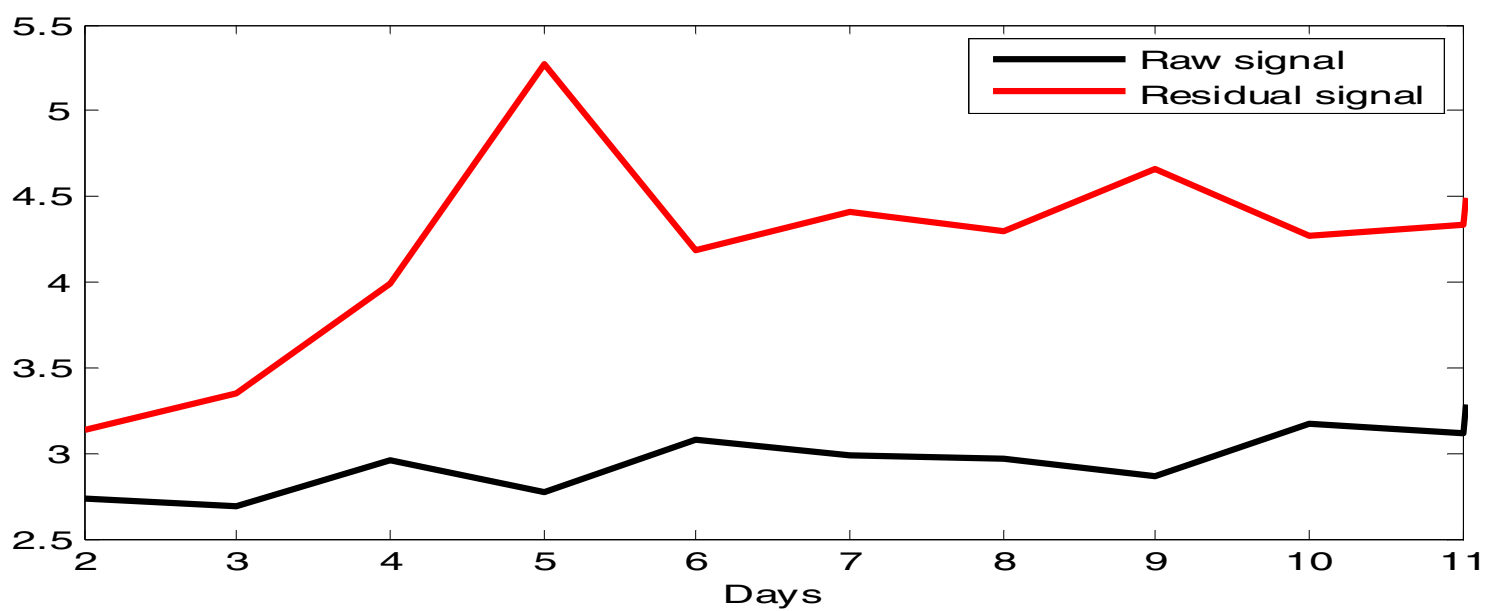

Fig. 14. Figure 13 zoomed.

method of EEMD and residual signal can be used to identify early damage in gear boxes. These results prove that the method can increase the precision of results given by the two methods EEMD and residual signal by reducing noise and preserving signal information.

Acknowledgements. The authors gratefully acknowledge LASPI (Laboratoire d'Analyse des Signaux et des Processus Industriels) for signals.

\section{References}

[1] B.D. Forrester, Use of Wigner Ville distribution in helicopter transmission fault detection in iProc of the Australian, symposium on signal Processing and Applications ASSPA89, Adelaide, Australia, 1989, pp. $77-82$

[2] D. Boulahbal, M.F. Golnaraghi, F. Ismail, Amplitude and phase wavelet maps for the detection of cracks in geared systems, Mech. Syst. Signal Process. 13 (1999) 423-436

[3] C. Capdessus, M. Sidahmed, Cyclostationary processes application in gear faults early diagnosis, Mech. Syst. Signal Process. 14 (2000) 371-685
[4] F. Bonnardot, M. El Badaoui, R.B. Randall, J. Danière, F. Guillet, Use of the acceleration signal of a gearbox in order to perform angular resampling (with limited speed fluctuation), Mech. Syst. Signal Process. 19 (2005) 766785

[5] Q. Gao, C. Duan, H. Fan, Q. Meng, Rotating machine fault diagnosis using empirical mode decomposition, Mech. Syst. Signal Process. 22 (2008) 1072-1081

[6] F. Combet, L. Gelman, Optimal filtering of gear signals for early damage detection based on the spectral Kurtosis, Mech. Syst. Signal Process. 23 (2009) 652-668

[7] N.E. Huang, Z. Shen, S.R. Long, The empirical mode decomposition and the Hilbert spectrum for nonlinear and non-stationary time series analysis, Proc. R. Soc. Lond. Ser. 454 (1998) 903-995

[8] N.E. Huang, Z. Shen, S.R. Long, A new view of nonlinear water waves: the Hilbert spectrum, Annu. Rev. Fluid Mech. 31 (1999) 417-457

[9] O. Andrade, S. Nasuto, P. Kyberd, C.M. Sweeney Reed, F.R.V. Kanijn, EMG signal filtering based on Empirical Mode Decomposition, Biomed. Signal Processing and Control 1 (2006) 44-55

[10] Xu Guanlei, Wang Xiaotong, Xu Xiaogang, Improved bidimensional EMD and Hilbert spectrum for the analysis of textures, Pattern Recogn. 42 (2009) 718-734 
[11] Rong Jiang, Hong Yan, Studies of spectral properties of short genes using the wavelet subspace Hilbert-Huang transform (WSHHT), Physica A 387 (2008) 4223-4247

[12] Xiaoli Li, Duan Li, Zhenhu Liang, Logan J. Voss, Jamie W. Sleigh, Analysis of depth of anesthesia with HilbertHuang spectral entropy, Clin. Neurophysiol. 119 (2008) $2465-2475$

[13] Jia-Rong Yeh, Shou-Zen Fan, Jiann-Shing Shieh, Human heart beat analysis using a modified algorithm of detrended fluctuation analysis based on empirical mode decomposition, Med. Eng. Phys. 31 (2009) 92-100

[14] S.J. Loutridis, Damage detection in gear systems using empirical mode decomposition, Eng. Struct. 26 (2004) 1833-1841

[15] B. Liu, S. Riemenschneider, Y. Xub, Gearbox fault diagnosis using empirical mode decomposition and Hilbert spectrum, Mech. Syst. Signal Process. 17 (2005) 1-17

[16] Z.K. Peng, P.W. Tse, F.L. Chu, A comparison study of improved Hilbert-Huang transform and wavelet transform: application to fault diagnosis for rolling bearing, Mech. Syst. Signal Process. 19 (2005) 974-988

[17] A. Parey, M. El Badaoui, F. Guillet, N. Tandon, Dynamic modeling of spur gear pair and application of empirical mode decomposition-based statistical analysis for early detection of localized tooth defect, J. Sound Vib. 294 (2006) 547-561

[18] H. Li, X. Deng, H. Dai, Structural damage detection using the combination method of EMD and wavelet analysis, Mech. Syst. Signal Process. 21 (2007) 298-306

[19] Yimin Shao, Fengshou Gu, Fazenda. B. Ball A, Luyang Guan, Gearbox fault diagnosis under different operating conditions based on time synchronous average and ensemble empirical mode decomposition, ICCAS-SICE, 2009, pp. 383-388

[20] Shufeng Ai, Hui Li, Gear Fault Detection Based on Ensemble Empirical Mode Decomposition and HilbertHuang Transform Fuzzy Systems and Knowledge Discovery, FSKD '08, 2008, Vol. 3, pp. 173-177

[21] P. Flandrin, G. Rilling, P. Goncalve, Empirical Mode Decomposition as a Filter Bank, IEEE Signal Process. Lett. 11 (2004) 112-114
[22] N.E. Huang, M.L. Wu, S.R. Long, A confidence limit for the empirical mode decomposition and Hilbert spectral analysis, Proc. R. Soc. Lond. 459 (2003) 2317-2345

[23] R.T. Rato, M.D. Ortigueira, A.G. Batista, On the HHT, its problems and some solutions, Mech. Syst. Signal Process. 22 (2008) 1374-1394

[24] Yanli Yang, Jiahao Deng, Caipeng Wu, Analysis of mode mixing phenomenon in the empirical mode decomposition method, Second Int. Symp. Inf. Sci. Eng. IEEE (2009) $553-556$

[25] Zhaohua Wu, N.E. Huang, Ensemble empirical mode decomposition: a noise-assisted data analysis method, advances in adaptive data analysis 1, 2009, 1-41 c world scientific publishing company

[26] R.M. Stewart, Some useful data analysis techniques for gearbox diagnosis. Applications of time series analysis, Ph.D. Thesis, ISVR, University of Southampton, 1977

[27] Siyan Wu, Ming J. Zuo, Anand Parey, Simulation of spur gear dynamics and estimation of fault growth, J. Sound Vib. 317 (2008) 608-624

[28] P.D. Mcfadden, Detecting fatigue cracks in gear by amplitude and phase demodulation of the meshing vibration, ASME Journal of vibration, Acoustics, Stress, and Reliability in Design 108 (1986) 165-170

[29] Enayet B. Halim, M.A.A. Shoukat Choudhury, Sirish L. Shah, Ming J. Zuo, Time domain averaging across all scales: A novel method for detection of gearbox faults, Mech. Sys. Signal Process. 22 (2008) 261-278

[30] M. El Badaoui, F. Guillet, J. Daniere, New applications of the real cepstrum to gear signals, including definition of a robust fault indicator, Mech. Sys. Signal Process. 18, (2004) 1031-1046

[31] L. Bouillaut, Approches cyclostationnaire et non linéaire pour l'analyse vibratoire de machines tournantes: Aspects théoriques et applications au diagnostic, Thèse Université de Technologie de Compiègne, 7 novembre 2000 